\title{
Systematic Analysis of Clinical Curative Effect of Acupuncture on Children with Cerebral Palsy Movement Disorder
}

\author{
Yujin Hou ${ }^{1, \mathrm{a}}$, Xiaosu Jie ${ }^{1, \mathrm{~b}}$, Xin Guo ${ }^{1, \mathrm{c}}$, Yingying Zhang ${ }^{1, \mathrm{~d}}$, Hua Shi ${ }^{1, \mathrm{e}}$, Yuanjun Lou 1, f \\ ${ }^{1}$ Henan Province Hospital of Traditional Chinese Medicine, Henan Zhengzhou, 450002
}

\begin{abstract}
Objective: To explore the effect of applying acupuncture on children with cerebral palsy dyskinesia. Methods: Research work was carried out in our hospital. The work was carried out from November 2018 to November 2019. A total of 100 patients were selected, all of whom were children with cerebral palsy, accompanied by dyskinesia. They were randomly divided into two groups, and were given conventional rehabilitation training and acupuncture treatment respectively, named the control group and the experimental group, and the clinical efficacy of the two groups of patients was analyzed. Results: There was no significant difference in the MAS scores of the two groups of children before treatment, which was expressed as $\mathrm{P}>0.05$. After treatment intervention, the scores of the two groups of children were improved, in contrast, the improvement effect is more significant for the experimental group, the data difference is large, $\mathrm{P}<0.05$, which is significant. The treatment effective rate of the children in the experimental group was $92.00 \%$, and the treatment effective rate of the children in the control group was $76.00 \%$, there is a big difference between the two groups of data, $\mathrm{P}<0.05$, which is significant. Conclusion: The application of acupuncture and moxibustion therapy in children with cerebral palsy movement disorders can improve the adverse symptoms of children and promote the recovery of patients, which has positive significance for clinical development.
\end{abstract}

\section{Introduction}

Pediatric cerebral palsy mainly refers to a type of non-progressive brain injury exhibited by the child from before birth to one month after birth. The child will show central never dyskinesia or abnormal posture. Patient children are usually accompanied by visual and auditory abnormalities, mental retardation, epilepsy and other behavioral disorders [1]. According to the results of relevant research data, the incidence of infantile cerebral palsy in China is relatively high, about $1.8 \%-4.0 \%$, which is the main motor disability of infants and young children. It is particularly important to give children early diagnosis and treatment. Acupuncture and moxibustion is a kind of physical therapy with high safety. This study is based on this, to explore the effect of acupuncture treatment in children with cerebral palsy movement disorders.

\section{Patient data and research methods}

\subsection{General information analysis}

The research work was carried out in our hospital. The work was carried out from November 2018 to November 2019. A total of 100 patients were selected, all of whom were children with cerebral palsy, accompanied by dyskinesia. They were randomly divided into two groups, and were given conventional rehabilitation training and acupuncture treatment respectively, named the control group and the experimental group, and the number of children in each group was 50 . The number of male and female children in the experimental group was 24 and 26 respectively. The average age of the children was (1.22 \pm 0.23 ) years old, including 21 cases of cesarean section and 29 cases of normal delivery. There are 4 examples of premature babies. In the control group, the number of males and females was both 25 , and the average age of the patients was $(1.54 \pm 0.55)$ years old. Among them, 3 cases were premature babies, 20 cases were cesarean section, and 30 cases were natural delivery. The difference in the general data of the two groups of children is small and can be compared.

\subsection{Research methods}

The children in the control group used conventional rehabilitation training. In the specific implementation process, each patient was under the responsibility of a professional doctor of Chinese medicine rehabilitation in our hospital, starting from the actual situation of the child, it develops targeted rehabilitation training, which mainly includes sitting posture training, head and neck rehabilitation training and limb training, etc. During the 
rehabilitation training given to the child, it is necessary to regularly check the child's thickness and movement, muscle tone, intelligence recovery, balancing situation, etc.

The children in the experimental group were also given acupuncture treatment while using rehabilitation training. First, as far as scalp acupuncture points are concerned, they mainly include Sishencong, Zhisanzheng, top midline, temporal three pins, top paraline and thread, etc. In the specific implementation process, it is necessary to first sterilize the local scalp of the child, select the acupuncture point and pierce it quickly at an angle of 30 degrees, and after the resistance of the needle decreases, reduce the angle of the needle, entering the needle by twisting it slowly, it needs to pay attention to avoid local bleeding in the child, or avoid the dizziness problem of the child. It should note that the needle retention time should be controlled at about 30 minutes. When pulling out the needle, ensuring that it is quickly pulled out, and then immediately give a cotton swab for local compression to prevent bleeding. The selection of acupuncture points for body acupuncture mainly includes tendon contraction, Neiguan, Weizhong, Zusanli, Huantiao, Renzhong, Xuanzhong, Baihui, Zusanli, Sishencong, Sanyinjiao and Yintang. Acupuncture points for upper extremities mainly include Hegu, Quchi and Jianyu. Acupuncture points on lower extremities mainly include Jiexi and Fumu. Acupuncture points on the upper and lower extremities need to be applied on the basis of conventional skin disinfection, and the 1.0-inch acupuncture needles should be applied. After the acupuncture has gained Qi energy, the needles should be left for 10-15 seconds, and then the needles should be discharged.

\subsection{Observation indicators}

The MAS scores of the two groups of children were compared, in which the score was high and the effect was

Table 1 Comparison of MAS scores between two groups of children before and after treatment $( \pm s)$

\begin{tabular}{ccc}
\hline Group & Before treatment & After treatment \\
\hline Experimental Group $(n=50)$ & $3.64 \pm 1.00$ & $2.04 \pm 0.22$ \\
Control Group $(n=50)$ & $3.55 \pm 1.25$ & $2.98 \pm 0.65$ \\
$t$ & 0.495 & 9.495 \\
P & $>0.05$ & $<0.05$ \\
\hline
\end{tabular}

\subsection{Treatment effective rate}

The treatment effective rate of the children in the experimental group was $92.00 \%$, and the treatment

Table 2 Comparison of treatment effect between two groups of children [n (\%)]

\begin{tabular}{|c|c|c|c|c|}
\hline Group & Marked Effect & Effective & Ineffective & Effective Rate \\
\hline $\begin{array}{c}\text { Experimental Group } \\
(n=50)\end{array}$ & $33(66.00)$ & $13(26.00)$ & $4(8.00)$ & $46(92.00)$ \\
\hline $\begin{array}{l}\text { Control Group } \\
\qquad(\mathbf{n}=\mathbf{5 0})\end{array}$ & $20(40.00)$ & $18(36.00)$ & $12(24.00)$ & $38(76.00)$ \\
\hline $\mathrm{X} 2$ & & & & 11.204 \\
\hline $\mathbf{P}$ & & & & $<0.05$ \\
\hline
\end{tabular}

effective rate of the children in the control group was

poor.

Comparing the treatment effects of the two groups of ildren, mainly including three indicators of marked marked effect means that: after the treatment, the child can walk independently, restore the motor function of the motor limbs, can realize the self-care of life, and other clinical symptoms can be improved or disappeared; Effective: The child's limb function has improved, other clinical symptoms have improved, however, they are unable to self-care of life and unable to walk independently. Ineffective: there was no significant change before and after treatment in patient child, and the condition even became worse. Excluding inefficiency is the total effectiveness rate of this study.

\subsection{Statistical methods}

Using the statistical software SPSS20.0 as a tool, performing statistical analysis on the data presented in this study, verifying the t-value of the comparison result of measurement data $( \pm s)$, and verifying the 2 value of the comparison result of counting data $(n, \%)$, when the result shows $\mathrm{P}<0.05$, it means that the difference between the groups has statistical analysis value [2].

\section{Results}

\subsection{MAS score comparison}

There was no significant difference in the MAS score of the two groups of children before treatment, which was expressed as $\mathrm{P}>0.05$. After the treatment intervention, the scores of the two groups of children were improved. The experimental group had a better result and was more significant, the data difference is large, $\mathrm{P}<0.05$, which is significant. $76.00 \%$. The data difference between the two groups was large, $\mathrm{P}<0.05$. which was significant, experimental group has a better result. 


\section{Discussion}

Judging from the current social development status, China's construction time has been strengthened, people's living environment and living habits have undergone major changes, and the incidence of cerebral palsy in children has also gradually increased, which has caused adverse effects on the normal growth and development of children, and even threatened children's life and health [3]. Pediatric cerebral palsy mainly refers to a type of non-progressive brain injury exhibited by the child from before birth to one month after birth. The child will show central dyskinesia or abnormal posture. Children are usually accompanied by visual and auditory abnormalities, mental retardation, epilepsy and other behavioral disorders. According to the results of relevant research data, the incidence of pediatric cerebral palsy in China is relatively high, about $1.8 \%-4.0 \%$, which is the main motor disability of infants and young children. It is particularly important to give children early diagnosis and treatment. From the perspective of traditional Chinese medicine, the analysis of the problem of cerebral palsy in children is considered to belong to the category of five hard, five late and five soft. It is caused by the damage of the marrow sea, patients often show qi and blood empty, insufficient heart and spleen, etc., it will cause damage to the heart, liver, brain and so on. Traditional Chinese medicine develops treatment work, mainly for patients to dredge meridians and promote meridian regeneration [4]. The application of acupuncture and moxibustion method is more effective. Among them, Baihui acupoint and Sishencong belong to the same acupuncture point in the human body, and giving them acupuncture treatment can improve the patient's yang energy, fill the sea of marrow, and have the effect of strengthening brain and intelligence. The Fengchi acupoints can play the role of removing wind. In the specific intervention process, it will be combined with Shenshu and Sanyinjiao to achieve the effect of regulating the liver and kidney, nourishing the veins and strengthening the bones. In the specific treatment process, the application of the warm meridian method can achieve a more significant meridian effect. If multiple methods are used in combination, the effect will be more significant. The results of this study showed that there was no significant difference in the MAS scores of the two groups of children before treatment, which was expressed as $\mathrm{P}>0.05$, it is meaningless. After treatment intervention, the scores of the two groups of children had both improved, in comparison, the score of the experimental group was more significant, the data difference is larger, $\mathrm{P}<0.05$, which is significant. The treatment effective rate of the children in the experimental group was $92.00 \%$, and the treatment effective rate of the children in the control group was $76.00 \%$.

When applying acupuncture and moxibustion to children with cerebral palsy dyskinesia, there are many types of methods, and the curative effect is more obvious. According to relevant data, most of the researchers choose acupuncture at the acupuncture point for point selection. It is shown that the application of acupuncture and moxibustion methods can improve patients' limb dysfunction, alleviate the muscle spasm of children, improve the muscle tone of children, improve joint activity, and have a significant application effect on the overall adjustment of children [5]. The basic disease of cerebral palsy in children is the brain, and the brain marrow is embedded in the head. It is a place for meridians, qi and blood to be collected for the organs. Intervention with scalp acupuncture can strengthen the blood flow of the brain of the child and stimulate the brain potential of the child to re-develop the brain and promote the reconstruction of brain function. At the same time, the apoptosis of brain cells is reduced, so that the nerve function of children can be restored. Therefore, when clinical treatment of pediatric cerebral palsy patients is carried out, scalp acupuncture should be the mainstay, and body acupuncture should be combined with it to promote the maximum application effect [6]. For the mechanism of acupuncture treatment of cerebral palsy, most of the clinical experiments are based on animal experiments, and the degree of in-depth understanding of the mechanism is not enough. It needs to be strengthened in future development. The results of the study show that the combined use of acupuncture treatment methods and various rehabilitation treatments can improve the independent living ability of the child and improve the degree of muscle spasm in the child. When combining acupuncture treatment methods with simple rehabilitation treatment or Comparing the drug treatment methods, the effect is extremely significant, indicating that acupuncture is effective in treating children with cerebral palsy, and it is better when used in combination with rehabilitation training and other methods. In actual development, due to factors such as complicated pathogenesis of children with cerebral palsy, there is still a lack of special drugs for the treatment of children with cerebral palsy, the acupuncture method can improve the patient's motor function and promote the patient's recovery, therefore, it can be used in clinical practice, combining it with comprehensive rehabilitation training and other methods [7], in order to form a comprehensive rehabilitation treatment method for children with cerebral palsy, so as to realize the sports awareness and social adaptability of children with cerebral palsy, and promote the recovery of children.

In summary, the application of acupuncture and moxibustion therapy in children with cerebral palsy movement disorders can improve the adverse symptoms of children and promote the recovery of patient children, which has a positive significance for clinical development.

\section{References}

1. Ma Yanju. Clinical and mechanism research of acupuncture combined with rehabilitation training in treatment of infantile cerebral palsy (spastic) [D]. Changchun University of Traditional Chinese Medicine, 2013.

2. Zhu Jing. Clinical Observation of Liu's Pediatric Massage and Acupuncture Treatment of Spastic 
Pediatric Cerebral Palsy [D]. Hunan University of Traditional Chinese Medicine, 2014.

3. Hong Lifei. Acupuncture treatment of cerebral palsy clinical efficacy and regulation of immune mechanism [D]. Guangzhou University of Traditional Chinese Medicine, 2010.

4. Shi Zhenguo. The clinical effect of electronic moxibustion Changqiang and Baihui acupoints combined with "Jin three acupuncture points" on children with cerebral palsy [D]. Fujian University of Traditional Chinese Medicine, 2019.

5. Hao Jing. Clinical study of acupuncture therapy combined with intellectual training in the treatment of mental retardation in children with cerebral palsy [D]. Shandong University of Traditional Chinese Medicine, 2010.

6. Chen Yihua. Clinical study on acupuncture treatment of cerebral palsy in children [D]. Guangzhou University of Traditional Chinese Medicine, 2007.

7. Zhang Xinxin. Observation on the therapeutic effect of electroacupuncture combined with traditional acupuncture on 46 children with cerebral palsy [J]. Chinese Journal of Practical Nervous Diseases, 2013,16 (17): 69-70. 